\title{
Internal Conversion and Vibrational Energy Redistribution in Chlorophyll A
}

Prathamesh M Shenai, ${ }^{\dagger}$ Sebastian Fernandez-Alberti,, ,‡ William P Bricker,, Sergei Tretiak, ${ }^{\S}$ and Yang Zhao*,†

$\dagger$ Division of Materials Science, Nanyang Technological University, Singapore 639798,

$\ddagger$ Universidad Nacional de Quilmes, Roque Saenz Pea 352, B1876BXD Bernal, Argentina,

|| Department of Energy, Environmental and Chemical Engineering, Washington

University, Saint Louis, Missouri 63130, USA, and

$\S$ Theoretical Division, Center for Nonlinear Studies (CNLS), and Center for Integrated

Nanotechnologies (CINT), Los Alamos National Laboratory, Los Alamos, New Mexico 87545, USA

E-mail: sfalberti@gmail.com; yzhao@ntu.edu.sg

${ }^{*}$ To whom correspondence should be addressed

${ }^{\dagger}$ Nanyang Technological University

$\ddagger$ Universidad Nacional de Quilmes

"Washington University

${ }^{\S}$ Los Alamos National Laboratory 
In the NA-ESMD simulations, the rescaling of nuclear velocities at the time of hop is taken to be in the direction of the non-adiabatic coupling vector as

$$
\dot{R}_{i}^{J}(t)=\dot{R}_{i}^{I}(t)-\gamma_{I J} d_{I J}^{i} / m_{i}
$$

for a hop from state $I$ to another state $J$, with $\gamma_{I J}$ defined as

$$
\gamma_{I J}=\frac{b_{I J} \pm \sqrt{b_{I J}^{2}+2 a_{I J}\left(E_{I}-E_{J}\right)}}{a_{I J}}
$$

where

$$
a_{I J}=\sum_{i}\left|d_{I J}^{i}\right|^{2} / m_{i}
$$

and

$$
b_{I J}=\sum_{i} \dot{R}_{i}^{I}(t) \cdot d_{I J}^{i}
$$

in order to maintain energy conservation. $E_{I}$ and $E_{J}$ denote the energies of adiabatic electronic states $I$ and $J$, respectively. This raise the possibility that the specifics of the normal modes analysis could be a consequence of the direct energy transfers that take place due to discrete jumps through the energy gaps between coupled excited-state potential energy surfaces. Therefore, the observed intramolecular vibrational energy redistribution could enter simulations only via rescaling of nuclear velocities in the direction of NACR vectors rather than as a consequence of the action of the nonadiabatic contribution to nuclear forces close to a crossing seam. In order to discern between these two possibilities, we have carried out simulations with a modified algorithm which rescales the atomic velocities in the same directions as they are at the time step before the hopping occurs. That is,

$$
\dot{R}_{i}^{J}(t)=\dot{R}_{i}^{I}(t)-\gamma_{I J} \dot{R}_{i}^{I}(t)
$$


with $\gamma_{I J}$ defined as

$$
\gamma_{I J}=1 \pm \sqrt{1+\left(E_{I}-E_{J}\right) / K_{I}}
$$

where

$$
K_{I}=\sum_{i} \frac{1}{2} m_{i}\left|\dot{R}_{i}^{I}(t)\right|^{2}
$$

is defined, as before, in order to satisfy the energy conservation during the $I$ to $J$ hop. All the initial conditions and simulation parameters are kept identical to those described in the main text. Fig. S1(a) shows the resulting dynamics of the excited state decay whereas Fig. S1 shows the total energies along the most important normal modes. The results obtained here, in terms of the rate constants as well as the identified active normal modes, are found to be unchanged as compared to the original results in the main text, thereby asserting their reliability.
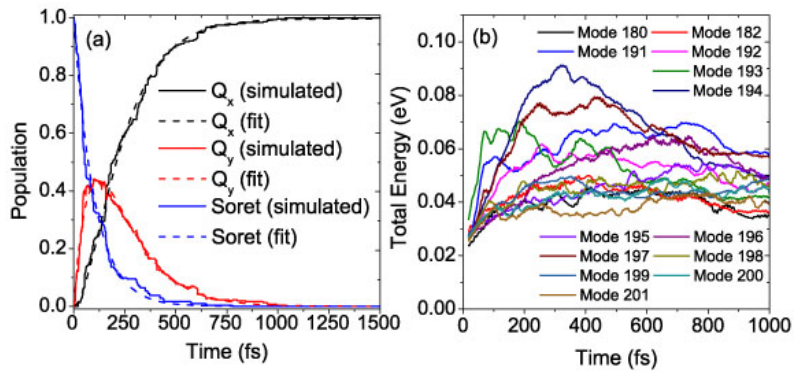

Figure S1: For excited state dynamics with modified algorithm to rescale velocities, (a) the population evolution on various excited states. The Soret band dynamics here takes into account both $S_{3}$ and $S_{4}$ states. (b) Running average of the total energy along normal modes 180, 182, 191-201. 